\title{
CONSISTENT PETROV-GALERKIN FINITE ELEMENT SIMULATION OF CHANNEL FLOWS
}

\author{
TONY W.H. SHEU* AND S.F. TSAI \\ Department of Naval Architecture and Ocean Engineering, National Taiwan University, 73 Chow-Shan Road, \\ Taipei, Taiwan, Republic of China
}

\begin{abstract}
SUMMARY
In this paper, Navier-Stokes fluid flows in curved channels are considered. Upstream of the backwardfacing step, there exists a channel with a $90^{\circ}$ bend and a fixed curvature of 2.5. The purpose of conducting this study was to apply a finite element code to study the effect of the distorted upstream velocity profile developing over the bend on laminar expansion flows behind the step. The size of the eddies formed downstream of the step is addressed. The present work employs primitive velocities, which stagger the pressure working variable, to assure satisfaction of the inf-sup stability condition. In quadratic elements, spatial derivatives are approximated within the consistent Petrov-Galerkin finite element framework. Use of this method aids stability in the sense that artificial damping is solely added to the direction parallel to the flow direction. Through analytical testing, in conjunction with two other benchmark tests, the integrity of applying the computer code in quadratic elements is verified. Copyright (C) 1999 John Wiley \& Sons, Ltd.
\end{abstract}

KEY WORDS: backward-facing step; quadratic elements; consistent Petrov-Galerkin

\section{INTRODUCTION}

Fluid flows in channels with flow reversals find many industrial applications. Typical examples are the flows in heat exchangers and ducts for industrial use, flows around buildings, and microelectric circuit boards. Among this class of flow problems, a channel with a backwardfacing step is regarded as having the simplest geometry while retaining rich flow physics manifested by the flow separation, flow reattachment and multiple recirculation bubbles in the channel. The above explains why expansion of a flow over a step has received a lot of attention in the literature and was selected for a benchmark study in this article.

Numerical prediction of flows in a backward-facing step has been the subject of intensive study over the last few decades. Much of the previous work has been directed towards studying the effects of the Reynolds number [1,2] and the expansion ratio on the flow development behind the step. Comparatively few studies have addressed the influence of the inlet flow on the downstream flow development in the channel [3,4]. This has prompted the current research into deepening the understanding about expansion flow behind a backward-facing step. We restrict ourselves to examining laminar flows to avoid the complication involved in applying turbulence models to equations of motion.

* Correspondence to: Department of Naval Architecture and Ocean Engineering, National Taiwan University, 73 Chow-Shan Road, Taipei, Taiwan, Republic of China. Fax: + 8862 23929885; e-mail: sheu@indy.na.ntu.edu.tw 
Only recently has the advent of powerful computational environments and the rapidly declining cost-to-performance ratio of computer hardware made possible the numerical study of Navier-Stokes fluid flows. Despite several decades of numerical experience, many obstacles still remain in obtaining a highly accurate flow simulation. Consideration will be given to the implementation of the outflow boundary condition and to approximation of equation nonlinearities.

In this paper, Section 2 presents a closure problem for the incompressible viscous fluid flow. Section 3 presents a discretization method that falls into the weighted residuals framework. An attribute of this upwind model is that both the pressure gradient and diffusive fluxes are weighted in favor of the upwind side. Attempts to better understand the proposed finite element model have prompted us to conduct a more theoretically appealing study. To achieve this goal, we conduct modified equation analysis on the linearized momentum as well as on the continuity equations. We will close the inflow-outflow problem by devising a free boundary condition. Section 4 tests the validity of the computer code against the analytical problem used for the study of incompressible flows. Then, a backward-facing step problem, a curved flow problem with a $90^{\circ}$ bend, and a channel flow problem with a bend and a step are analyzed. Finally, Section 5 offers conclusions.

\section{MODEL EQUATIONS}

Newtonian fluids that are subject to the incompressibility constraint condition are considered. The Navier-Stokes equations along with the divergence-free constraint equation constitute the working equations. For convenience of analysis, these equations are cast in the following dimensionless form:

$$
\begin{aligned}
& \underline{u} \cdot \nabla \underline{u}=-\nabla p+\frac{1}{R e} \nabla^{2} \underline{u}, \\
& \nabla \cdot \underline{u}=0 .
\end{aligned}
$$

In the above elliptical-differential system, $R e=u_{0} L / v$ denotes the Reynolds number, $L$ is the characteristic length, $u_{0}$ is the characteristic velocity, and $v$ is the kinematic viscosity of the fluid. Applied to incompressible fluid flows, the above velocity-pressure formulation has been proven to be desirable because this variable setting accommodates closure conditions $[5,6]$.

To complete the specification of the elliptical problem, it is necessary that boundary conditions are prescribed on the boundary of the physical domain. For this reason, either velocities at $\Gamma_{\mathrm{D}}$,

$$
\underline{u}=\underline{g}
$$

or the Neumann-type boundary condition on the open boundary $\Gamma_{\mathrm{N}}$, are specified, with a unit outward normal vector $\underline{n}$,

$$
-p \underline{n}+\frac{1}{\operatorname{Re}} \frac{\partial \underline{u}}{\partial n}=f_{\text {traction }}
$$




\section{THEORETICAL FORMULATION}

Having reached a high degree of mathematical sophistication, finite element methods (FEM) are now the typical numerical technique of choice. Among the formulations, the present authors are in favor of the mixed formulation for use in the present study of incompressible fluid flow. The main reason making the mixed formulation appealing to use is that the kinematic constraint of a divergence-free vector field is unconditionally ensured.

The variational statement characterizing the conservation equations (1) and (2) is as follows: For an admissive function $\underline{w} \in H_{0}^{1}(\Omega) \times H_{0}^{1}(\Omega)$ and a pressure mode $q \in L^{2}(\Omega) / R=P$, find weak solutions for velocity-pressure $(\underline{u}, p) \in V \equiv\left(H_{0}^{1} \times H_{0}^{1}\right) \times P$ from the following equations:

$$
\begin{aligned}
& \int_{\Omega}(\underline{u} \cdot \nabla \underline{u}) \cdot \underline{w} \mathrm{~d} \underline{x}+\frac{1}{R e} \int_{\Omega} \nabla \underline{u}: \nabla \underline{w} \mathrm{~d} \underline{x}+\int_{\Omega} p \nabla \cdot \underline{w} \mathrm{~d} \underline{x}=\int_{\Omega} f \cdot \underline{w} \mathrm{~d} \underline{x}, \quad \forall \underline{w} \in H, \\
& \int_{\Omega}(\nabla \cdot \underline{u}) q \mathrm{~d} \underline{x}=0, \quad \forall q \in P .
\end{aligned}
$$

\subsection{Quadratic streamline upwind Petrov-Galerkin (SUPG) finite element model}

In circumstances when convection largely dominated its diffusion counterpart, employment of an upwind model is essential to compensate for the potential loss of numerical stability. In this study, the Petrov-Galerkin method is adopted, where the test functions $\underline{w}$ used in Equation (5) are regarded as upwind-weighted refinements to the basis functions. This upwinding procedure, while providing non-oscillatory velocities, may cause the prediction accuracy to deteriorate owing to false diffusion errors added to the formulation $[7,8]$. Such cross-wind diffusion errors are particularly pronounced in cases when grid lines and flow directions are not in a good alignment. To alleviate the degree of obscured real physics for problems under these circumstances, a flow-oriented upwind model is adopted as a remedy to stabilize the discrete equation without the cost of deteriorating the prediction accuracy along the flow direction. Attempts to improve solution accuracy motivated the use of higher-order elements. In what follows, the analysis is performed in a domain covered by quadratic elements.

The key to suppressing oscillatory pressures is the appropriate selection of finite elements. To avoid oscillatory modes contaminating the solution, it is necessary that finite elements must satisfy the inf-sup stability condition $[9,10]$. In order for the predicted solutions to be smooth, the shape functions for $N(\underline{x})$ (for the velocity vector) and $M(\underline{x})$ (for the pressure) are chosen to be biquadratic and bilinear polynomials respectively. By substituting the chosen test and basis equations into Equations (5) and (6), the finite element matrix equations can be derived. The resulting matrix equation is

$$
\left[\int_{\Omega^{h}}\left\{\begin{array}{ccc}
C^{i j} & 0 & -M^{j} \frac{\partial N^{i}}{\partial X_{1}}+B^{i} \frac{\partial M^{j}}{\partial X_{1}} \\
0 & C^{i j} & -M^{j} \frac{\partial N^{i}}{\partial X_{2}}+B^{i} \frac{\partial M^{j}}{\partial X_{2}} \\
M^{i} \frac{\partial N^{j}}{\partial X_{1}} & M^{i} \frac{\partial N^{j}}{\partial X_{2}} & 0
\end{array}\right] \mathrm{d} \Omega^{h}\right]\left(\begin{array}{c}
u_{j} \\
v_{j} \\
p_{j}
\end{array}\right]=\underline{0},
$$

where 


$$
B^{i}=\tau N^{j} \tilde{V}_{k}^{j} \frac{\partial N^{i}}{\partial x_{k}},
$$

and

$$
C^{i j}=\left(N^{i}+B^{i}\right) N^{j} \tilde{V}_{k}^{j} \frac{\partial N^{j}}{\partial x_{k}}+\frac{1}{R e} \frac{\partial N^{i}}{\partial x_{k}} \frac{\partial N^{j}}{\partial x_{k}}-\frac{1}{R e} B^{i} \frac{\partial^{2} N^{j}}{\partial x_{k} \partial x_{k}} .
$$

It is sufficient to state that $\tilde{V}_{k}^{j}$ are assumed to be constants when formulating the matrices. These values are evaluated through a simple arithmetic mean using the two most updated velocities. Having derived the above indefinite and unsymmetric matrix equations, it is necessary to define $\tau$ to complete the finite element formulation. Depending on the nodal classification, $\tau$ can be derived as

$$
\tau\left(\gamma_{\xi}\right)=\left\{\begin{array}{ll}
\alpha\left(h_{\mathrm{rl}}, \gamma_{1}\right) ; & \text { at end-nodes } \\
\beta(\gamma) ; & \text { at center-nodes }
\end{array},\right.
$$

where

$$
\begin{aligned}
& \alpha\left(h_{\mathrm{r} 1}, \gamma_{1}\right)=\left[-\left(h_{\mathrm{r} 1}+h_{\mathrm{r} 1} \gamma_{1}\right) \mathrm{e}^{-2 \gamma 1}+4\left(2 h_{\mathrm{r} 1}+h_{\mathrm{r} 1} \gamma_{1}\right) \mathrm{e}^{-\gamma 1}-7\left(h_{\mathrm{r} 1}+1\right)+4\left(2-h_{\mathrm{r} 1} \gamma_{1}\right) \mathrm{e}^{h_{\mathrm{r}} \gamma_{1}}\right. \\
& \left.-\left(1-h_{\mathrm{r} 1} \gamma_{1}\right) \mathrm{e}^{2 h_{\mathrm{r} 1} \gamma_{1}}\right] \\
& /\left[-\left(6 h_{\mathrm{r} 1}-h_{\mathrm{r} 1} \gamma_{1}\right) \mathrm{e}^{-2 \gamma_{1}}+4\left(3 h_{\mathrm{rl}}-2 h_{\mathrm{r} 1} \gamma_{1}\right) \mathrm{e}^{-\gamma_{1}}+14 h_{\mathrm{r} 1} \gamma_{1}+6\left(-h_{\mathrm{r} 1}+1\right)\right. \\
& \left.-4\left(3+2 h_{\mathrm{r} 1} \gamma_{1}\right) \mathrm{e}^{h_{\mathrm{r} 1} \gamma_{1}}+\left(6+h_{\mathrm{r} 1} \gamma_{1}\right) \mathrm{e}^{2 h_{\mathrm{r} 1} \gamma_{1}}\right], \\
& \beta(\gamma)=\frac{1}{2} \operatorname{coth}\left(\frac{\gamma}{2}\right)-\frac{1}{\gamma}, \\
& h_{\mathrm{r} 1}=h_{\mathrm{r}} / h_{1}, \\
& \gamma_{1}=u_{\mathrm{e}} h_{1} \operatorname{Re} / 2 \text {, } \\
& \gamma=u_{\mathrm{c}} h \operatorname{Re} / 2 \text {. }
\end{aligned}
$$

For the sake of completeness, Figure 1 plots the value of $\tau$ against the Peclet numbers in the uniform grid limiting case. As is evident from Equations (9) and (10), the formulation generalizes the scheme of Donea on uniform grids [11],

$$
\tau_{\text {Donea }}(\gamma)= \begin{cases}\alpha_{\text {Donea }}(\gamma)=\frac{2-\cosh (\gamma)-\frac{4}{7} \tanh \left(\frac{\gamma}{2}\right)+\frac{1}{\gamma} \sinh (\gamma)}{4 \tanh \left(\frac{\gamma}{2}\right)-\sinh (\gamma)-\frac{6}{\gamma} \sinh (\gamma) \tanh \left(\frac{\gamma}{2}\right)} ; & \text { at end-nodes, } \\ \beta(\gamma)=\frac{1}{2} \operatorname{coth}\left(\frac{\gamma}{2}\right)-\frac{1}{\gamma} ; & \text { at center-nodes. }\end{cases}
$$

\subsection{Fundamental study of the finite element model}

Much of the intricate detail of the finite element model can be inferred from the modified equation analysis. In this light, we are motivated to conduct this analysis by use of the MAPLE [12] to get rid of much of the algebraic complexities in the modified equation analysis conducted on biquadratic finite elements. Following the standard procedure in conducting the modified equation analysis, the modified continuity equation is obtained as 


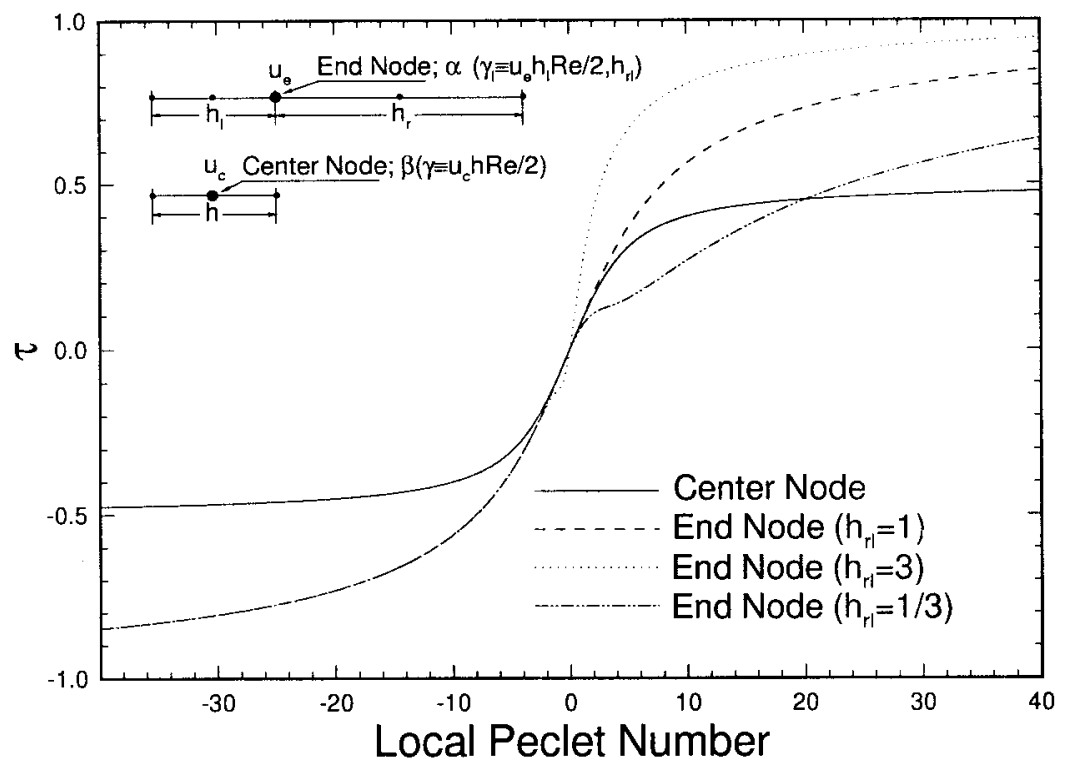

Figure 1. The values of $\tau$, as given in Equations (9) and (10), against Peclet numbers for the proposed upwind finite element model.

$$
\begin{aligned}
u_{x}+v_{y}= & -\left(\frac{h^{2}}{3} u_{x x x}+\frac{h^{2}}{3} u_{x y y}+\frac{h^{4}}{20} u_{x x x x x}+\frac{h^{4}}{9} u_{x x x y y}+\frac{h^{4}}{36} u_{x y y y y}\right) \\
& -\left(\frac{h^{2}}{3} v_{x x y}+\frac{h^{2}}{3} v_{y y y}+\frac{h^{4}}{36} v_{x x x x y}+\frac{h^{4}}{9} v_{x x y y y}+\frac{h^{4}}{20} v_{y y y y y}\right)+\mathcal{O}\left(h^{6}\right) .
\end{aligned}
$$

To this point we have shown evidence which reveals that Equation (12) approaches the desired divergence-free state with an order of $\mathcal{O}\left(h^{2}\right)$.

A Taylor series expansion is similarly applied to linearized momentum equations, resulting in their differential counterparts. At the representative node, marked with a $\boldsymbol{O}$ in Figure 2 , the modified equations for the $x$ and $y$ momentum equations are obtained respectively as

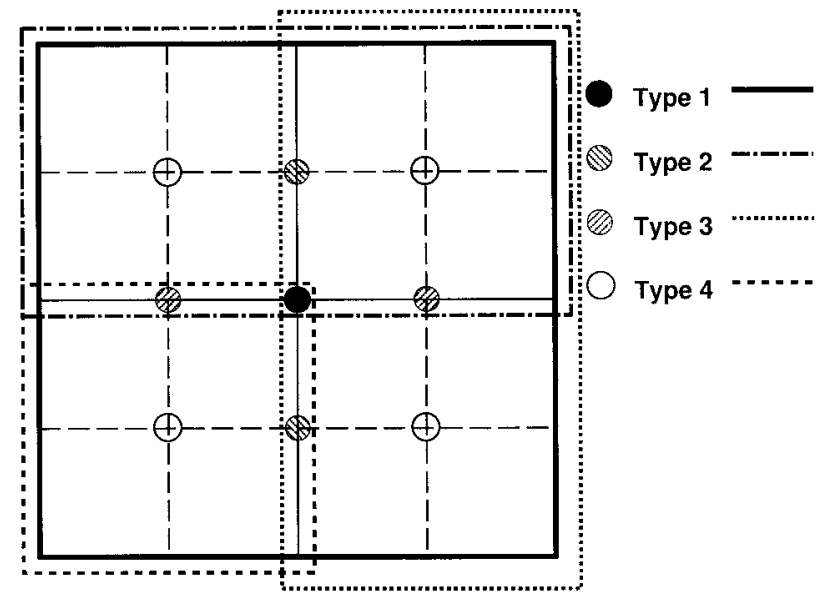

Figure 2. An illustration of four basic elements for the point labeled with

in a quadratic element. 


$$
\begin{aligned}
& a u_{x}+b u_{y}+p_{x}-\frac{1}{R e}\left(u_{x x}+u_{y y}\right)-f_{1}=\left(a^{2}+b^{2}\right) \tau u_{s s}+D_{u}+P_{u}, \\
& a v_{x}+b v_{y}+p_{y}-\frac{1}{R e}\left(v_{x x}+v_{y y}\right)-f_{2}=\left(a^{2}+b^{2}\right) \tau v_{s s}+D_{v}+P_{v} .
\end{aligned}
$$

In the above two equations, the subscript $s$ denotes the streamline direction. The discretization errors for the flux terms, $D_{u}$ and $D_{v}$, and pressure gradients, $P_{u}$ and $P_{v}$, are summarized in Tables I and II respectively. In the light of Equations (13) and (14), the conclusion is that the scheme has been recognized as being able to stabilize the discrete equation owing to damping terms added along the flow direction. By taking the positive velocities $a$ and $b$, shown in Equations (13) and (14), as an example, it is expected that physically relevant solutions will be obtained provided that the value of $\tau$ is positive.

Table I. Discretization errors $D_{i}(i=u, v)$, as shown in Equations (13) and (14), for four types of elements in Figure 2

\begin{tabular}{llllll}
\hline $\begin{array}{l}\text { Coefficient element } \\
\text { type }\end{array}$ & $u_{x x x}$ or $v_{x x x}$ & $u_{x x y}$ or $v_{x x y}$ & $u_{x y y}$ or $v_{x y y}$ & $u_{y y y}$ or $v_{y y y}$ \\
\hline Type 1 & $\frac{1}{3} \frac{a\left(-9 \tau+h^{2} R e\right)}{R e}$ & $\frac{1}{5} \frac{b\left(-5 \tau+h^{2} R e\right)}{R e}$ & $\frac{1}{5} \frac{a\left(-5 \tau+h^{2} R e\right)}{R e}$ & $\frac{1}{3} \frac{b\left(-9 \tau+h^{2} R e\right)}{R e}$ \\
Type 2 & $\frac{1}{3} \frac{a\left(-9 \tau+h^{2} R e\right)}{R e}$ & $\frac{1}{5} \frac{b\left(-5 \tau+h^{2} R e\right)}{R e}$ & $-\frac{1}{10} \frac{a\left(10 \tau+h^{2} R e\right)}{R e}$ & $-\frac{1}{6} b h^{2}$ \\
Type 3 & $-\frac{1}{6} a h^{2}$ & $-\frac{1}{10} \frac{b\left(10 \tau+h^{2} R e\right)}{R e}$ & $\frac{1}{5} \frac{a\left(-5 \tau+h^{2} R e\right)}{R e}$ & $\frac{1}{3} \frac{b\left(-9 \tau+h^{2} R e\right)}{R e}$ \\
Type 4 & $-\frac{1}{6} a h^{2}$ & $-\frac{1}{10} \frac{b\left(10 \tau+h^{2} R e\right)}{R e}$ & $-\frac{1}{10} \frac{a\left(10 \tau+h^{2} R e\right)}{R e}$ & $-\frac{1}{6} b h^{2}$ \\
\hline
\end{tabular}

Table II. Discretization errors $P_{i}(i=u, v)$, as shown in Equations (13) and (14), for four types of elements, as given in Figure 2

\begin{tabular}{lcclllll}
\hline \multicolumn{1}{c}{$p_{x x}$} & $p_{x y}$ & $p_{y y}$ & $p_{x x x}$ & $p_{x x y}$ & $p_{x y y}$ & $p_{y y y}$ \\
\hline Equation (13) & & & & & & \\
Type 1 & $3 \tau a$ & $\tau b$ & 0 & $-\frac{2}{3} h^{2}$ & 0 & 0 & 0 \\
Type 2 & $3 \tau a$ & $\tau b$ & 0 & $-\frac{3}{3} h^{2}$ & 0 & $-\frac{1}{2} h^{2}$ & 0 \\
Type 3 & 0 & $\tau b$ & 0 & $-\frac{1}{9} h^{2}$ & 0 & 0 & 0 \\
Type 4 & 0 & $\tau b$ & 0 & $-\frac{1}{6} h^{2}$ & 0 & $-\frac{1}{2} h^{2}$ & 0 \\
Equation (14) & & & & & & \\
Type 1 & 0 & $\tau a$ & $3 \tau b$ & 0 & 0 & 0 & $-\frac{2}{3} h^{2}$ \\
Type 2 & 0 & $\tau a$ & 0 & 0 & 0 & 0 & $-\frac{1}{9} h^{2}$ \\
Type 3 & 0 & $\tau a$ & $3 \tau b$ & 0 & $-\frac{1}{2} h^{2}$ & 0 & $-\frac{3}{3} h^{2}$ \\
Type 4 & 0 & $\tau a$ & 0 & 0 & $-\frac{1}{2} h^{2}$ & 0 & $-\frac{1}{6} h^{2}$ \\
\hline
\end{tabular}




\subsection{Implementation of outflow boundary condition}

For a closure reason, one can apply physical arguments at a solid wall or a free surface to prescribe the boundary conditions. This is not the case for the specification of boundary condition at the outlet because no physical reasoning is available for us to achieve this goal. Despite years of research, the difficulty of specifying a permissible condition at the truncated boundary has not been fully resolved. This difficulty is particularly pronounced for a simulation that involves a solid boundary to which a boundary layer is attached. Under this circumstance, the artificial boundary should be far away from the inlet. This helps prevent unphysical feedback noises, emanating from the outflow boundary, from being propagated upstream and destroying the solution. Unfortunately, the analysis domain must be truncated somewhere; otherwise, the disk storage and computer time requirements may be greatly in excess of that provided by computers of the day. Thus, there exists a trade-off between these two considerations.

In this paper, the so-called free boundary condition approach is used together with the present mixed finite element formulation [13]. There is no specification of an ad hoc stress boundary condition at the artificial boundary. The direct consequence of applying the free boundary condition to the finite element formulation is that the surface traction vector $f_{\text {traction }}$ is one part of the solution to be computed. It is noteworthy that the underlying boundary condition matches the physics of fluid flows inside the domain. As a consequence, both non-linear and diffusive fluxes in the flow can be taken into account.

\section{COMPUTED RESULTS}

As a first step towards the assessment of the simulation quality, we consider a problem that is amenable to an analytical solution. With this in mind, it is preferable to consider problems with smooth and simple flow patterns. Our argument is as follows:

If a discretization scheme fails to accurately predict a simple problem, then there must be plenty of room for further improvement of the scheme. In the numerical simulation of smooth flows, a good way for justifying the computer code is to cast the computed errors in their $L_{2}$-norm form. To answer whether or not the stability property is attainable, we conduct the rate of convergence study. This study proceeds with computing finite element errors $\left\|e_{1}\right\|$ and $\left\|e_{2}\right\|$ from two consecutive grids with grid sizes of $h_{1}$ and $h_{2}$. The rate of convergence is then obtained as

$$
c=\frac{\ln \left\|e_{1}\right\|-\ln \left\|e_{2}\right\|}{\ln \left|h_{1}\right|-\ln \left|h_{2}\right|} .
$$

The validation of the code is followed by considering an analytical problem in a square. The domain under investigation is confirmed in $0 \leq x, y \leq 1$, within which it is covered with uniform grids. The analytical pressure is obtained as

$$
p=\frac{-2}{(1+x)^{2}+(1+y)^{2}},
$$

provided that the boundary velocities are analytically specified as follows:

$$
u=\frac{-2(1+y)}{(1+x)^{2}+(1+y)^{2}},
$$


Table III. Computed errors and rates of convergent order

\begin{tabular}{cllll}
\hline Mesh size & $\left\|\underline{u}-\underline{u}_{\text {exact }}\right\|$ & $\begin{array}{l}\text { Rate of convergent } \\
\text { order } c\end{array}$ & $\left\|p-p_{\text {exact }}\right\|$ & $\begin{array}{l}\text { Rate of convergent } \\
\text { order } c\end{array}$ \\
\hline $5 \times 5$ & $1.374 \times 10^{-3}$ & & $2.404 \times 10^{-3}$ & \\
$10 \times 10$ & $3.757 \times 10^{-4}$ & 1.871 & $6.085 \times 10^{-4}$ & 1.982 \\
$20 \times 20$ & $8.817 \times 10^{-5}$ & 2.091 & $1.421 \times 10^{-4}$ & 2.098 \\
$40 \times 40$ & $1.863 \times 10^{-5}$ & 2.242 & $3.446 \times 10^{-5}$ & 2.044 \\
\hline
\end{tabular}

$$
v=\frac{2(1+x)}{(1+x)^{2}+(1+y)^{2}} .
$$

According to Table III, which tabulates the $L_{2}$-error norms, the integrity of the fully weighted SUPG finite element code is analytically validated.

\subsection{Benchmark test-a backward facing step problem}

The flow over a backward-facing step is the prototype best-suited for justifying the use of our proposed free boundary condition at the synthetic boundary. The wide spread popularity of this problem is attributable to two factors. First, there exists well-documented experimental and numerical data for this problem. Second, the flow physics are far from simple, even in a channel with simple geometry. It is the geometric simplicity of the problem that facilitates computation, comparison among schemes, and drives our study of this problem. As seen in Figure 3, the aspect ratio of the height of the backward-facing step, $s$, to the width of the cross-sectional, $H$, is chosen to be $s: H=1: 2$.

We impose at the channel inlet, which is upstream of the step with a distance of $H$, a fully developed flow. No-slip conditions are imposed at solid walls. In the present study, we consider a Reynolds number $R e=800$, which is obtained by considering the velocity $\bar{u}=\frac{2}{3} U_{\max }$ and the length $H$ as reference quantities. Our previous studies revealed that as $L / s>32$, analysis data become reliable since the traction forces come fairly close to zero [13]. Results presented here were those for a case with length $L$ at 32 .

We denote $x_{1}$ as the computed reattachment length of the recirculating zone behind the step. We also define $x_{4}$ as the separation length and $x_{5}$ as the reattachment length of the secondary recirculation bubble on the roof of the channel. These values are plotted in Figure 4 and are compared with the finite element results of Sohn [14], the finite difference results of Durst and Pereira [2], and the experimental data of Armaly et al. [1]. The reattachment length obtained here compares very favorable with the experimental results for smaller Reynolds number cases. Increasing $R e$ further causes the eddy to grow. At $R e=400$, the agreement of computed reattachment lengths with those obtained from the experimental measurement breaks down in that the flow becomes three-dimensional.

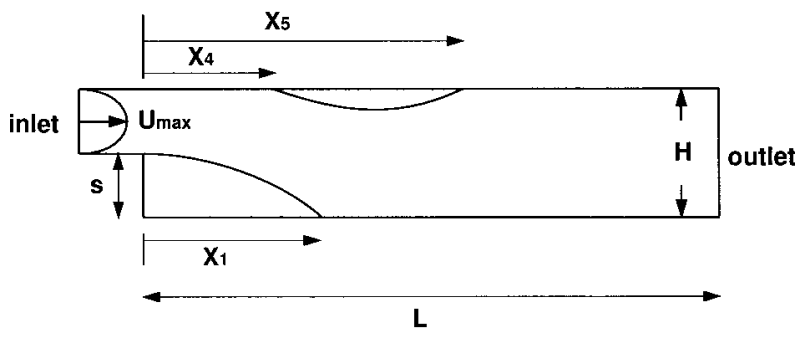

Figure 3. Backward-facing step geometry. 


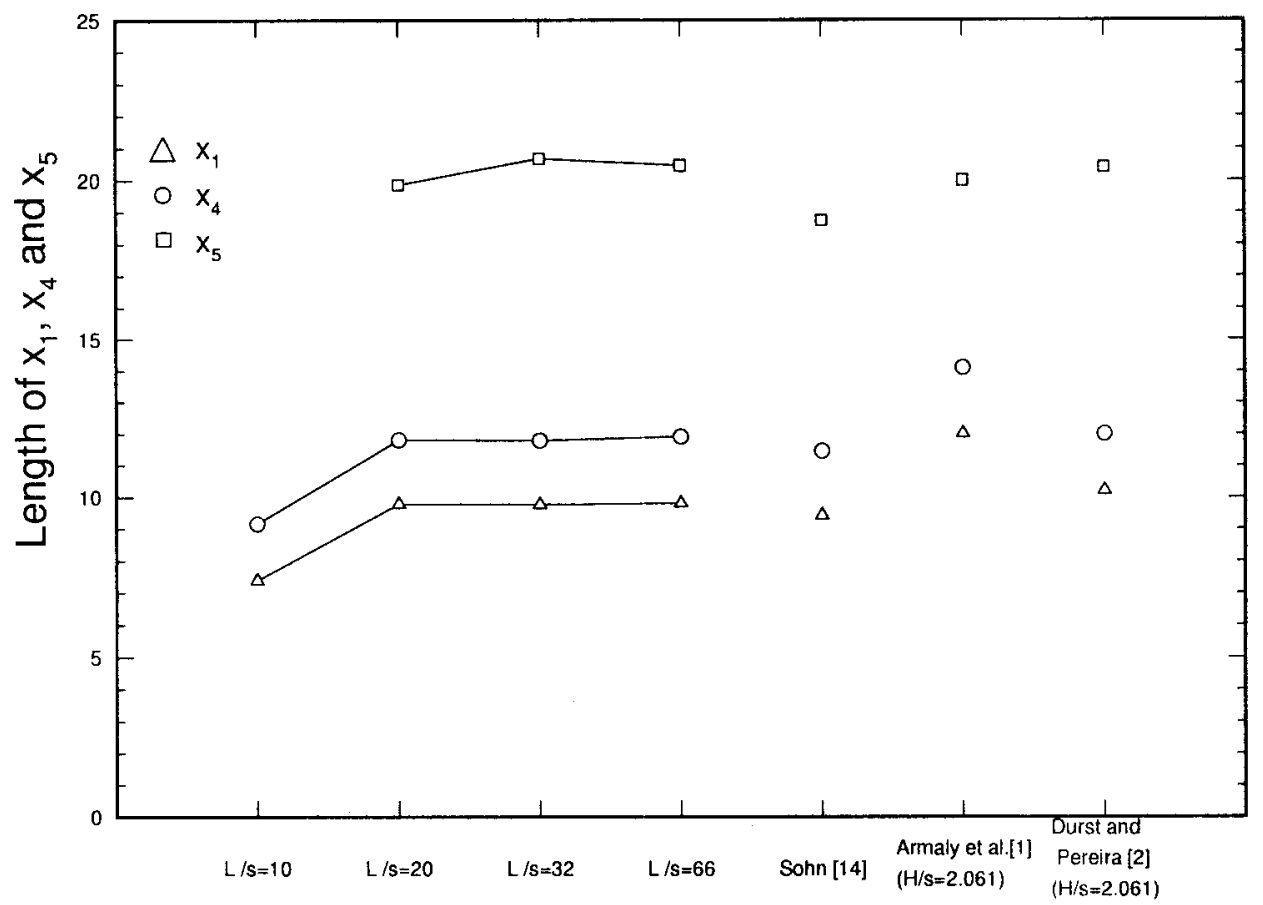

Figure 4. The computed reattachment lengths $x_{1}, x_{5}$ and the separation length $x_{4}$ against different values of $L / s$.

\subsection{Flow in a duct with a smooth $90^{\circ}$ bend}

There are two reasons why bend flows have received much attention in the literature. From the physical standpoint, centrifugal force plays an essential role for a flow into a curved duct. Such a centrifugal force causes a flow distortion to occur and the distorted velocity profile, in turn, influences the pressure distribution. Moreover, the motion in a curved channel is susceptible to instability when the destabilizing centrifugal force exceeds the restoring normal pressure gradient. On computational grounds, the bend under investigation is algebraically describable, thus simplifying mesh generation and facilitating the computation. On top of this, plenty of well-documented data are easily accessible in the literature for conducting comparison studies.

How the flow develops in a curved duct depends on the radius ratio of the duct and the Reynolds number. In this study, we simulated the flow development in a duct shown schematically in Figure 5. The curved section is characterized as having a curvature of 2.3 $\left[\equiv\left(r_{i}+0.5 D\right) / D\right]$. The flow under investigation had straight extension upstream and downstream of lengths that are five times the hydraulic diameter $D(=40 \mathrm{~mm})$. Based on the mean inflow velocity, the hydraulic diameter of the duct, and the viscosity $\left(\mu=1.98 \times 10^{-2}\right)$, the Reynolds number was 790. This is the benchmark exercise for the experimental condition of Humphrey et al. [15]. Flow instability caused by significant curvature effects was not reported in the experiment. Numerical results are presented for a grid with a resolution of $128 \times 64$, which was believed to be adequate to resolve the fine structure of this flow.

According to the computed velocity profiles plotted in Figure 6, the fluid flow in the straight section manifests itself by the boundary layer formation and a potential core region with blockage effects. What is most apparent in the pressure distribution plotted in Figure 7 is that 


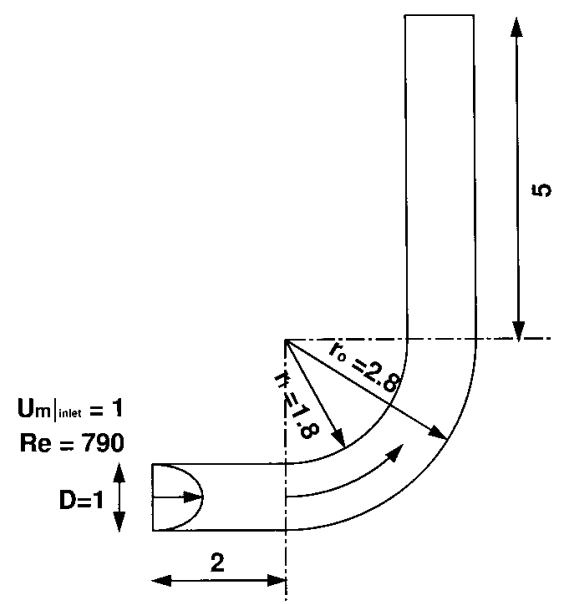

Figure 5. An illustration of the flow in a duct with a smooth $90^{\circ}$ bend and a constant curvature of 2.3 .

the flow inside the channel evolves into acceleration in regions near the inner radius wall due to the favorable longitudinal pressure gradient. Conversely, a slight deceleration occurs in regions near the outer radius wall because of the adverse pressure gradient. According to Figure 8 , which plots the pressure along the inner and outer radius walls, there is evidence referring to the outer bend as the pressure side, while the inner bend as the suction side. This figure reveals that the pressure gradient is strongly adverse over the inner (suction) surface for about $45^{\circ}$, while it is strongly favorable over the outer (pressure) bend for the same region.

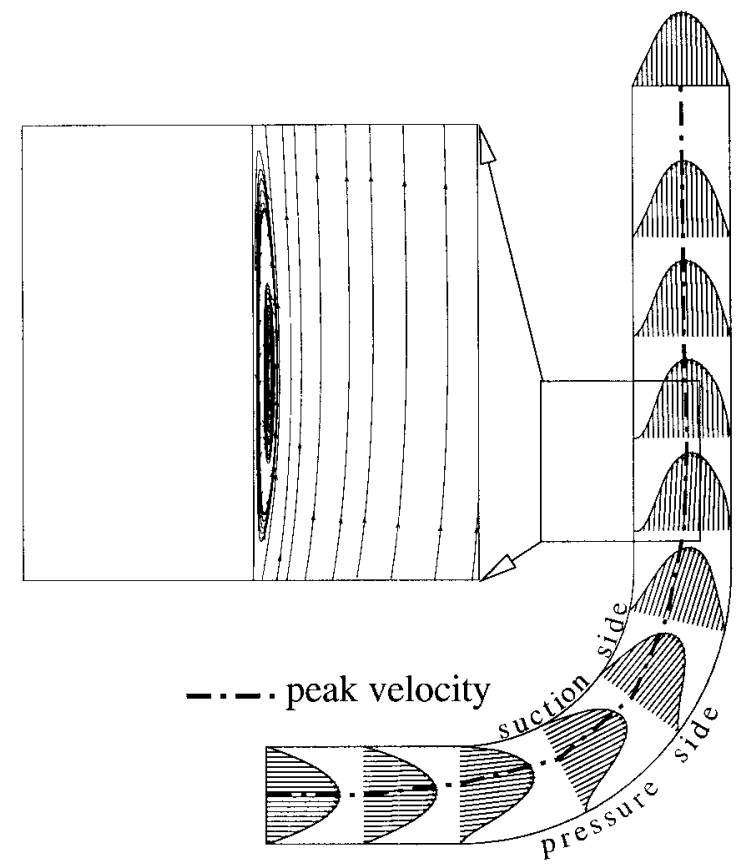

Figure 6. Velocity vector plots in the curved channel and the close-up plot of streamlines in the region behind the $90^{\circ}$ bend. 


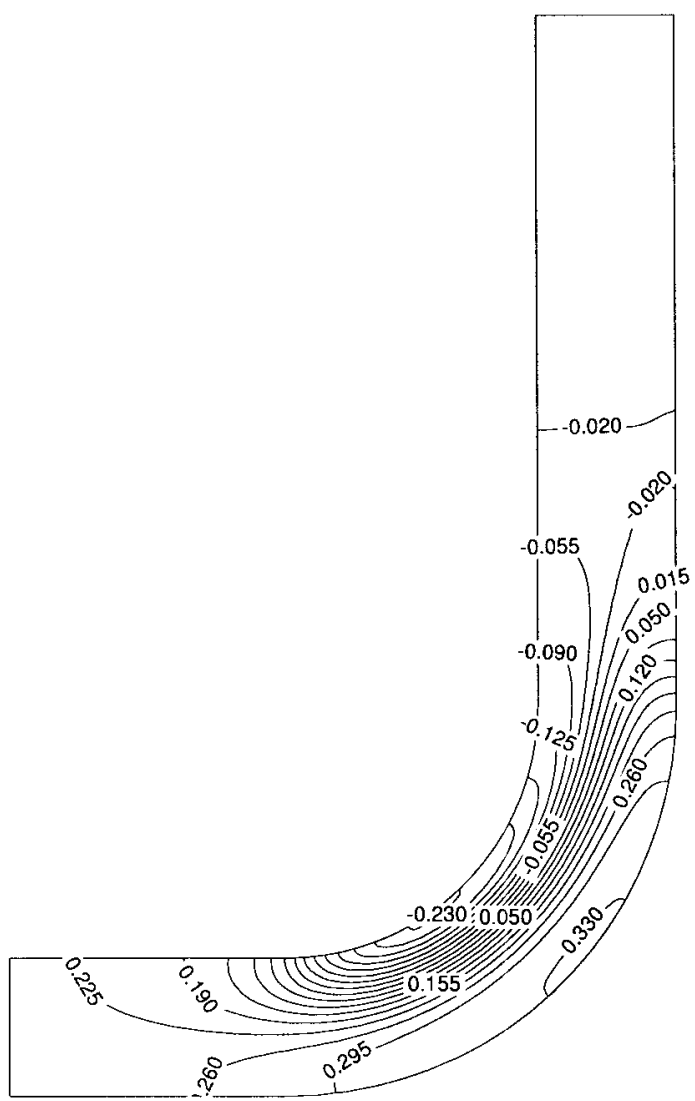

Figure 7. The computed pressure contours in the curved channel.

One characteristic of the flow structure in the bend is that velocities of smaller magnitude are observed in regions adjacent to the pressure surface.

As the velocity profiles plotted in Figure 6 show, the spatial location where the velocity has a peak value moves towards the spatial location closer to the suction side of the bend. Downstream of the bend, the streamwise velocity decelerates in regions near the suction side of the duct. This is followed by gradual development into a fully developed flow.

\subsection{Flow in a channel with a bend and a backward-facing step}

Having described successfully simulations of two benchmark tests in Sections 4.1 and 4.2, we will consider an even more difficult channel flow problem. This problem, shown in Figure 9, involves an upstream bend of fixed curvature 2.5 and a downstream backward-facing step. This problem is chosen to study the effect of the upstream inlet flow on the downstream flow separation behind the step.

The underlying idea of placing a $90^{\circ}$ bend is to construct a distorted velocity profile upstream of the step. Our argument is that progressive development is accompanied by acceleration of the flow in regions near the inner radius wall due to the favorable longitudinal pressure gradient, and that, conversely, there is flow deceleration around the outer radius region because of an adverse pressure gradient. Depending on the downstream length behind 


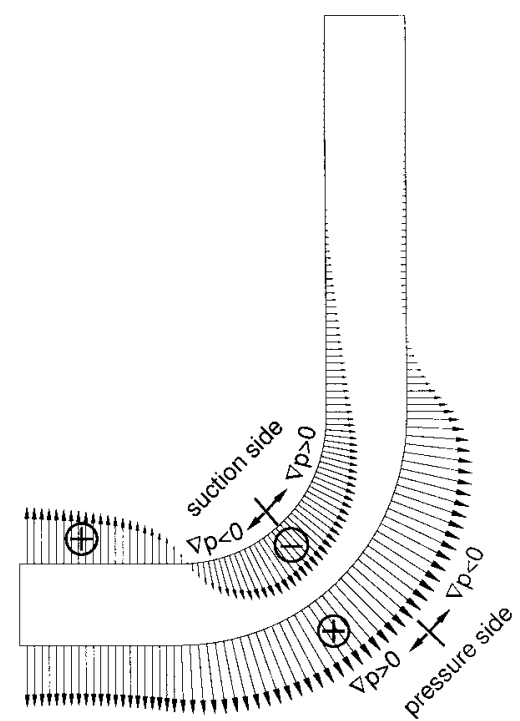

Figure 8. Pressure distributions along inner and outer curved walls.

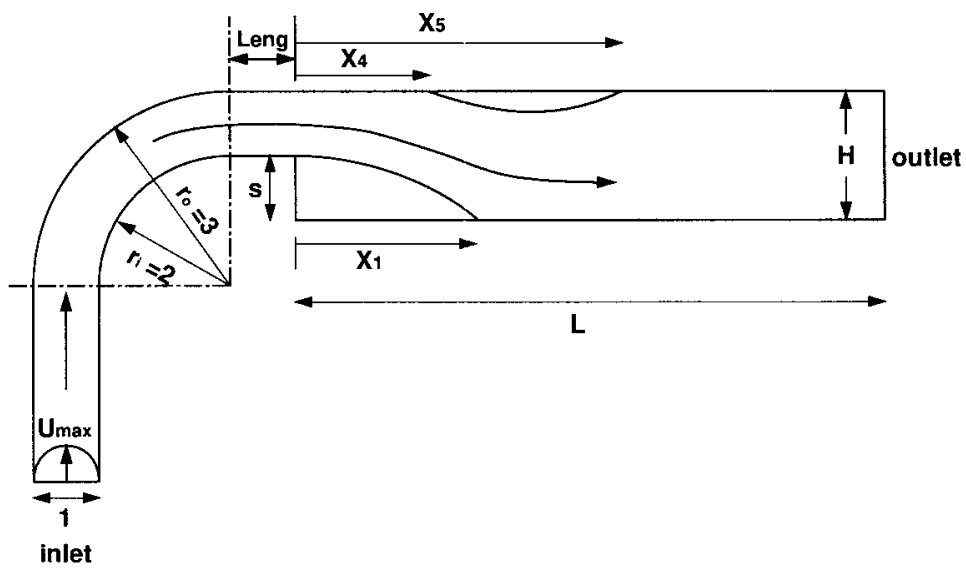

Figure 9. An illustration of the problem considered in Section 4.3.

the bend, different degrees of velocity distortion become apparent. As a consequence, we vary the length 'Leng', as seen in the Figure 9, and plot velocity profiles at several selected streamwise sections in Figure 10. The resulting reattachment and separation lengths, labeled $x_{1}, x_{4}, x_{5}$, are tabulated in Table IV.

\section{CONCLUSIONS}

The main thrust of this work has been to evaluate the potential of our upwind finite element model for the simulation of incompressible Navier-Stokes equations, subject to outflow boundary conditions, in steady state. The basis for the method adopted here is the use of a fully-weighted upwinding finite element model, which means that convective, diffusive and 


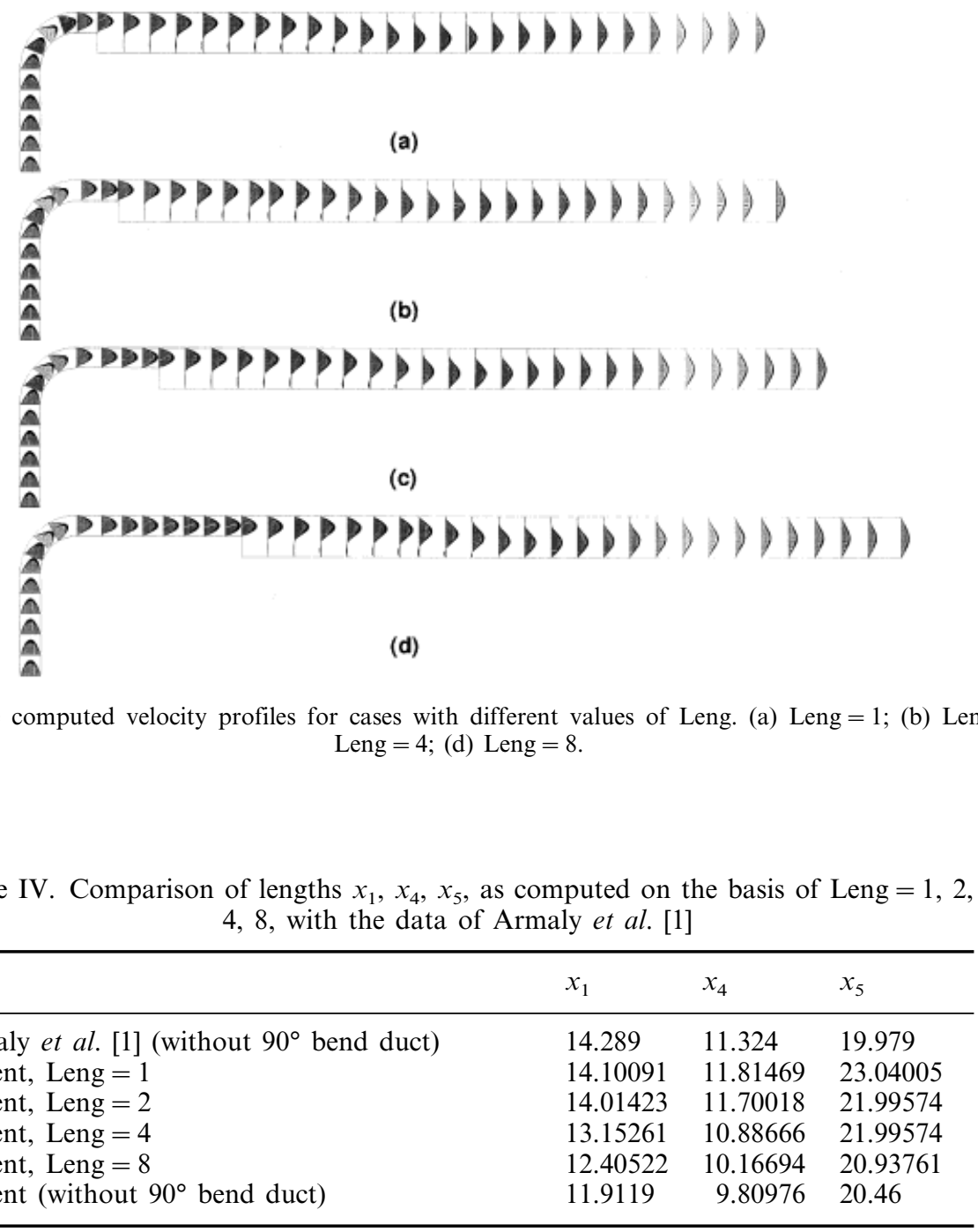

The length Leng is given in Figure 9.

pressure gradient terms are all biased-weighted. The proposed model is known as a streamline upwind finite element model, as evidenced by the modified equation analysis, which has provided insight into the method formulated in quadratic elements. The developed finite element model has been applied to the through-flow problem, together with the use of a free boundary condition approach. This method is featured as taking nodal values of $u, v$ and $p$ at the outlet as unknowns, which are coupled with the interior unknowns through surface integrals in the momentum equations. The results obtained for the steady flow case have been analytically verified. The justification for using the proposed finite element model to simulate channel flows has been presented further through several benchmark tests. 


\section{REFERENCES}

1. B.F. Armaly, F. Durst, J.C.F. Pereira and B. Schonung, 'Experimental and theoretical investigation of backwardfacing step flow', J. Fluid Mech., 127, 473-496 (1983).

2. F. Durst and J.C.F. Pereira, 'Time-dependent laminar backward-facing step flow in a two-dimensional duct', $J$. Fluids Eng., 110, 289-296 (1988).

3. Z. Qin and M.D. Olson, 'Finite element-algebraic closure analysis of turbulent separated-reattaching flow around a rectangular body', Comput. Methods Appl. Mech. Eng., 85, 131-150 (1991).

4. Z. Qin and M.D. Olson, 'Finite element-algebraic closure modelling of turbulent separated flow over a backward-facing step: steady and unsteady aspects', Int. J. Numer. Methods Heat Fluid Flow, 2, 3-20 (1992).

5. M.D. Gunzburger, Finite Element Methods for Viscous Incompressible Flows, A Guide to Theory, Practice, and Algorithms, Academic Press, New York, 1989.

6. O. Ladyzhenskaya, The Mathematical Theory of Viscous Incompressible Flow, Gordon and Breach, New York, 1969.

7. B.P. Leonard, 'A stable and accurate convective modelling procedure based on quadratic upstream interpolation', Comput. Methods Appl. Mech. Eng., 19, 59-98 (1979).

8. G.D. Raithby, 'Skew upwind differencing schemes for problems involving fluid flow', Comput. Methods Appl. Mech. Eng., 9, 153-164 (1976).

9. F. Brezzi, 'On the existence, uniqueness and approximation of saddle point problems arising from Lagrangian multipliers', RAIRO Anal. Numer., 8, 129-151 (1974).

10. I. Babuška, 'Error bounds for finite element methods', Numer. Math., 16, 322-333 (1971).

11. J. Donea, T. Belytschko and P. Smolinski, 'A generalized Galerkin method for steady convection-diffusion problems with application to quadratic shape function elements', Comput. Methods Appl. Mech. Eng., 48, 25-43 (1985).

12. B.W. Char, K.O. Geddes, G.H. Gonnet, B. Leong, M.B. Morgan and S.M. Watt, First Leaves: A Tutorial Introduction to MAPLE V, Springer, New York, 1992.

13. M.T. Wang and T.W.H. Sheu, 'Implementation of a free boundary condition to Navier-Stokes equations', Int. J. Numer. Methods Heat Fluid Flow, 7, 95-111 (1997).

14. J.L. Sohn, 'Evaluation of FIDAP on some classical laminar and turbulent benchmarks', Int. J. Numer. Methods Fluids, 8, 1469-1490 (1988).

15. J.A.C. Humphrey, A.M.K. Taylor and J.H. Whitelaw, 'Laminar flow in a square duct of strong curvature', $J$. Fluid Mech., 83, 509-527 (1977). 